\title{
Research on China English Teaching Based on Multi-Elements Analysis
}

\author{
Xiaokun Liu ${ }^{1, \text { a }}$ Mouqing Wang ${ }^{1, b^{*}}$ Zongyuan $\operatorname{Tan}^{2, \mathrm{c}}$ and Jian Zhou ${ }^{2, \mathrm{~d}}$ \\ ${ }^{1}$ School of Foreign Languages, Northwest Minzu University, Lanzhou, China \\ ${ }^{2}$ College of Electrical Engineering, Northwest Minzu University, Lanzhou, China \\ a614252705@qq.com, bwmqking@126.com, 'tanzongyuansmile@163.com \\ dzhoujianfrank@126.com
}

*The corresponding author: Wang Mouqing (wmqking@126.com)

\section{Keywords: English teaching; Training method; Multi-elements}

\begin{abstract}
The main purpose of this paper is to explore how the training method in English teaching is affected by the language cultural differences between Chinese and English. We focus on the analysis the pros and cons of education system in China and America. For instance, the basic expression of language, the sentence structure, and differences of pragmatics would have a great influence for impacting future language education. Moreover, according to the analysis on actual data, we find that similar to the education model of the combination of Chinese and English should be widely applied into future English teaching. Overall, we hope our analysis and proposal could as a reference for enhancing the quality of future English teaching.
\end{abstract}

\section{Introduction and Setting}

In some sense, China's English teaching is actually an international language teaching, rather than the general foreign language teaching [1]. The English perhaps deemed as an official or most popular language when the people attempt to cognize the foreign culture [2]. Furthermore, with the increasing enhancement of China's international status, Chinese culture has been focused by more and more scholars. Chinese culture can be regarded as the most significant component in cross-cultural communication. Of note, we also find that certain advanced western culture can be potentially regarded as a direction for reforming China's English education [3]. Education is a potential mean of affecting human behavior, which runs through social development [4]. The educational system of China is mainly follow the way that is from the outside to the inside to educate individual and uses external force for demanding individual to follow designated planning study, which can contribute to quickly grasp basic knowledge. The aim of China's education is that it has stronger durability, satisfies with the current situation and makes progress while maintaining stability. However, it is lack of practice innovative capability and isn't conducive to cultivate imagination.

\section{Rhetorical Method}

Metaphor is a rhetorical method, which is very common method of language expression in Chinese. China is an Ethnic diversity country and it is good at using the animal feature as certain character between each minority [5,7]. It could make language expression more image through this method. A great number of people also have large differences for the understand of metaphor because of the differences of cultural environment between Chinese and English. In China culture, there are representative instances to testify above statements. For example, dragon is the totem of Chinaman and the symbol of imperial power. The sons of the heaven were treated as the descendants of the Dragon were treated as the children of China [8]. Thus, ordinary people have such good wish that they hope their children to be dragon. In contrast, many countries which use English as mother 
language regard dragon as monster and the token of evil. At the same time, the wide spread wolves in China folk are the pronoun of ungratefulness. However, American regard snakes as the pronoun of returning kindness with evil. It is to show that the differences of language expression are suffer from the impact of metaphor $[9,10]$.

In addition, the expression meaning in certain color also have differences because of the differences of cultural background of language between Chinese and English. Though China and America use red to denote the celebration or happy days, and red also have different expression meaning. For instance, "to see red" expresses the implication that makes one's anger, and "wave a red flag" denotes an unhappy thing to result in a bad mood. The expression of Chinese language can't simply follow the meaning of the word and must understand the background. For example, an idiom translates into "glowing with health" and it express happy meaning, which don't demand to directly translate red and has the red implication. It can reveal that comprehending the background knowledge is very significant for language expression. Therefore, rhetorical method of Chinese denotes different culture connotation [11]. On the whole, assume that English sentences don't give any context, and also don't make any explanation, which merely know the English expression meaning and is hard to make corresponding imagination. Chinese is different from English, which can almost imagine different things by the simple language or vocabulary. Different languages all show such phenomenon, namely, they have particular way of appellation and usage rules, which decide the communication ways between each other. people are fond of using name to be directly called each other when they are talking, regardless of the relationship between relatives and friends.

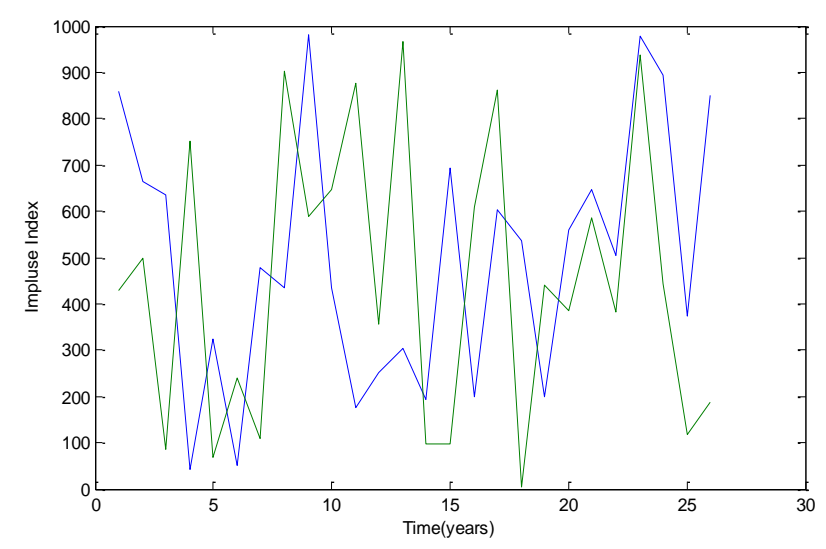

Figure 1. The relation of time and impulse index

\section{Form of Apology}

Even Homer sometimes nods. When we make mistakes, and always want to seek a suitable way to apologize for solving each misunderstand. The form of knowing how to apologize and finding out the most reasonable ways of apology, which will be a mean to effectively settle problem and they are the important component of politeness for language. The methods of apology have a great diversity of way in China and America, which is hard to select the proper apology way with respect to people who are lack of apology knowledge. Meanwhile, the form of apology has certain constraint conditions regardless of Chinese or English, which can't simply use language to apologize or forget to consider a reasonable way of apology. Thus, it is very necessary to comprehend the apology differences between China and America. For instance, when we accept others apology in China, and usually say that it doesn't matter. Nevertheless, the English expression "it doesn't matter" can't denote the fact that someone accept others apology, and we should select another suitable sentence, namely "that's all right, that's ok and no problem".

In consequence, if we don't understand the differences between Chinese and English and naturally follow the thinking habits of Chinese language to select corresponding English sentence 
for apology, which will result in misunderstand between each other. What's worse, the bad habits for apology could accompany us in the lifetime. When we talk to others, the communication of each other is not merely restricted to language, and might transmit information by using facial expression, gestures and other movements of the body. For example, the simple way that smile stretch out one hand can denote welcome.

At the same time, frown can express dissatisfaction in general situation, and on the contrary, nod can denote satisfaction. The way of this basic movements to communication of non-verbal have widely accepted between China and America, which is constantly used in society. For example, many people like to use the pattern of appellation for the use of name, which is "appellation plus name". However, there aren't the form in the English expression, but such way of appellation is used by Chinese. People are easily subject to the impact of a thinking habit, which is keen on named each other's name in Chinese way. It results in the fact that many people feel uncertain for using name way to name someone, and will increase the learning difficulty for learner. In China, it could be directly called name between familiar and intimate persons, but in America, this way of appellation does not show such phenomenon that people always have very familiar or intimate relationship. There are prominent differences in word order between Chinese and English, which profoundly reflect the fact that different national culture and thinking modes between Chinese and English have an effect on language. It is the main reason that resulting in the formation of word order is the thinking mode and folkways between Chinese and English. On the one hand, the research of the differences of word order can better contribute to master the language discrepancies. On the other hand, the study of different thinking mode is conducive to grasp the differences of word order. Firstly, English pays attention to the structural form of subject and predicate, which have fixed form that is "subject plus verb" except imperative sentences.

However, Chinese sentences lays emphasis on the expression of themes, in which can generally contain any structural sentence. In the first place, it can have theme and subject in the Chinese sentences, which have such formation that the theme is in front and the subject is behind. In the next place, the expression of English must have subject, which is to prove the fact that the sentences accord with English expression, but Chinese expression can omit subject and not follow general Chinese expression. In the end, Chinese can omit subject or theme in the special environment, but subject must exist in the English sentences. For example, a sentence is "have you buy tickets?". Secondly, in the Chinese, adverbial modifiers can be placed between the subject and predicate. However, in the English, adverbial modifiers generally are placed in the empennage of sentences and only frequency adverbs are placed between subject and predicate. Finally, the place of attribute of Chinese is in front of the central noun, but attribute of English can be placed in front or behind of noun. In summary, the differences of word order between Chinese and English mainly reflect the differences of thinking habits of China and America.

Education is a potential mean of affecting human behavior, which runs through social development. The English teaching of China is mainly follow the way that is from the outside to the inside to educate individual and uses external force for demanding individual to follow designated planning study, which can contribute to quickly grasp basic knowledge. The aim of China's education is that it has stronger durability, satisfies with the current situation and makes progress while maintaining stability. However, it is lack of practice innovative capability and isn't conducive to cultivate imagination. In contrast, the teaching of America is the educational concept of people oriented, namely, people break through shackles of current education culture by new and old culture to pursue the world concept of free and advances with the times. In addition, the educational system of America attaches great importance to the cultivation of independent analysis and practice capability, which advocates liberty of thought and guides independent thinking. It also lays emphasis on the importance of innovation and encourages independent or cooperation innovation. Thus, the American education can facilitate the full development of individuality by its way.

The differences between Chinese and English affect the development of China English teaching. It is very necessary to understand the purpose of English teaching between China and America, 
which can gradually eliminate educational differences, and contributes to fully make comparisons and research in the teaching. This way can discard the dross and extract the essence, which further facilitate the language fusion of China and America. As we all know, the differences of Chinese and English are objective existence. Only if put the purpose of teaching between China and America as foothold, actively develop all kinds of communication platform on Chinese and English and pay attention to the differences could reach the education purpose that cultivate more and more outstanding talents. At the same time, the differences of Chinese and English bring about serious impact for China English teaching, and only combine the differences of Chinese and English with the purpose of China and America teaching, which can contribute to the development of China teaching all the time.

\section{Conclusions}

The main purpose of this article is to explore how the training method in English teaching is affected by the language cultural differences between Chinese and English.

In fact, with the increasing revolution of China education system, in particular foreign language teaching, the advanced model and concept innovation of teaching and application have been focused by more and more relevant researcher. Based on the above comparison analysis, we find that the future English education model in China should combine the differences and characteristics between Chinese and English. In this regard, certain corresponding strategies for differences of language of Chinese and English are presented in Section 3, such as the education original purpose and the teaching pattern and model. In summary, we hope our analysis on language differences between Chinese and English could provide a pathway, which would act as a direction for further improving and enhancing the quality of English teaching in China.

\section{Acknowledgements}

This work is supported by Scientific Research Innovation Subject of Northwest Minzu University (Grant NO. Yxm2016082). This work is supported by Fundamental Research Funds for the Central Universities (Grant 31920160003), and by Research Funds for New Silk Road Economic Belt of Northwest Minzu University (Grant xscz1201602).

\section{References}

[1] J. X. Xi, M. He, H. Liu and J. F. Zheng: J Franklin Inst, Vol. 353 (2016) No.16, pp.4074-4090.

[2] N. Cai, J. W. Cao, H. Y. Ma and C. X. Wang: Arab J Sci Eng, Vol. 39 (2014) No.3, pp. 2427-2434.

[3] Y. Q. Guan, Z. J. Ji, L. Zhang and L. Wang: Int. J. Robust Nonlin. Control, DOI: 10.1002/rnc.3798.

[4] C. Xia, L. Wang, S. Sun and J. Wang: Nonlinear Dynamics, Vol. 69 (2012) No.3, pp.927-934.

[5] Z. J. Ji, H. Lin and H. S. Yu: IEEE Trans. Automat. Control, Vol. 60 (2015) No.3, pp.781-786.

[6] S. Iyer, T. Killingback, B. Sundaram and Z. Wang: PLoS ONE, Vol. 8 (2013) No.4, pp.1-17.

[7] N. Cai, M. J. Khan: Int. J. Control Automat. Syst., Vol. 15 (2017) No.1, pp.169-177.

[8] N. Cai, C. Diao and M. J. Khan: Complexity, 4978613, 2017.

[9] Z. J. Ji and H. S. Yu: IEEE Trans. Cybernet., DOI: 10.1109/TCYB.2016.2549034.

[10] J. Wu, M. Barahona, Y. J. Tan and H. Z. Deng: IEEE Trans. on Systems, Man, and Cybernetics - Part A: Systems and Humans, Vol. 41 (2011) No.6, pp.1244-1252.

[11]N. Cai, M. He, Q. Wu and M. J. Khan: J. Syst. Sci. Complexity, DOI: 10.1007/s11424-017-6273-7. 